\title{
Balkanologie
}

Balkanologie Revue d'études pluridisciplinaires

Vol. IV, $n^{\circ} 1 \mid 2000$

Volume IV Numéro 1

\section{Retour sur l'affaire macédonienne : une approche psycho-sociale}

\section{Nikos Kalampalikis}

\section{(2) OpenEdition}

1 Journals

\section{Édition électronique}

URL : http://journals.openedition.org/balkanologie/768

DOI : $10.4000 /$ balkanologie.768

ISSN : 1965-0582

Éditeur

Association française d'études sur les Balkans (Afebalk)

Édition imprimée

Date de publication : 1 septembre 2000

ISSN : 1279-7952

\section{Référence électronique}

Nikos Kalampalikis, "Retour sur l'affaire macédonienne : une approche psycho-sociale », Balkanologie [En ligne], Vol. IV, nº 1 | 2000, mis en ligne le 29 juillet 2010, consulté le 17 décembre 2020. URL

http://journals.openedition.org/balkanologie/768 ; DOI : https://doi.org/10.4000/balkanologie.768

Ce document a été généré automatiquement le 17 décembre 2020.

(c) Tous droits réservés 


\title{
Retour sur l'affaire macédonienne : une approche psycho-sociale
}

\author{
Nikos Kalampalikis
}

\section{Le scandale d'une prophétie}

1 Une bonne partie des Grecs d'aujourd'hui doit sûrement se souvenir d'une déclaration étrange prononcée en 1993 par K. Mitsotakis, Premier ministre grec de droite de l'époque: «dans dix ans l'affaire skopjienne sera oubliée ». Cette parole considérée comme étonnante sinon scandaleuse, véritable "trahison nationale", par une bonne partie du monde politique, de l'opinion publique et de la presse au moment où elle fut prononcée semble avoir eu en elle quelque chose de prophétique vue sous un prisme actuel. Destin curieux pour une déclaration considérée comme une insulte au sentiment collectif d'un peuple révolté, de même qu'une affaire ayant pris les proportions d'un "vol de l'âme grecque". Destin tragique pour son énonciateur, qui en a perdu son poste et pour son gouvernement, le pouvoir.

2 Loin de nous la volonté de prendre parti politiquement en nous référant à cette phrase. Seul son contenu nous intéresse. Avant de l'évoquer, revenons un peu sur l'ambiance qui régnait en Grèce pendant ces années, afin que les lecteurs puissent se faire une idée, de ce que signifiait véritablement l'affaire macédonienne (ou "skopjienne") à ce moment précis.

\section{L'affaire macédonienne (1991-1999)}

3 C'est au tout début des années 1990, quand les Républiques qui composent la fédération yougoslave se déchirent, que la version moderne de l'affaire macédonienne débute. À l'aube de la guerre, une des six Républiques yougoslaves, devient indépendante et autonome, sous le nom de "République de Macédoine", grâce à un référendum recueillant $95 \%$ de voix positives (8 septembre 1991).

4 Sa superficie est égale à celle de la Bretagne française et sa population compte deux millions d'habitants. Quelques mois plus tard (juillet 1992), elle choisit comme drapeau 
une étoile, symbole de la dynastie des anciens Macédoniens de l'époque d'Alexandre le Grand. Sa constitution fait allusion à des territoires macédoniens "occupés" et à des "minorités slavo-macédoniennes" vivant dans le reste des Balkans.

5 Soulignons au passage que la région macédonienne, espace géographique jamais strictement délimité depuis des siècles, et théâtre du conflit, appartient depuis la fin des guerres balkaniques (Traité de Bucarest, 1913), pour une moitié à la Grèce, et pour l'autre à la République yougoslave et à la Bulgarie'.

6 La Grèce réagit instantanément par voie diplomatique (4 décembre 1991) à cette nouvelle situation socio-politique qui prend corps à ses frontières. Au départ, avant l'adoption du drapeau, sa réaction était concentrée sur trois points : le nom du nouvel État, sa constitution (notamment le premier paragraphe de l'article $49^{2}$ ) et sa propagande qui lui était hostile. Elle considérait que le nom de "Macédoine", qui appartient également à l'une de ses provinces septentrionales, faisait partie uniquement de son propre héritage culturel et historique. De plus, une propagande hostile et certains articles de la constitution voisine semblaient menacer potentiellement son intégrité territoriale.

7 La prise en compte de ce différend par ses partenaires européens se manifeste dans un paragraphe distinct de la décision de la Commission européenne au sujet de la nouvelle situation en ex-Yougoslavie, fin 1991 (17 décembre 1991). Ce paragraphe mentionne que les nouvelles républiques ex-yougoslaves doivent garantir et établir "des conditions de sécurité et de respect des frontières en évitant des activités de propagande hostile envers un pays communautaire voisin, y compris une appellation supposant des revendications territoriales $»^{3}$.

8 Les deux premières garanties de cette décision (frontières, propagande) justifiaient presque pleinement deux des trois points au cœur de l'opposition grecque. Mais la troisième, celle du nom, malgré son caractère restrictif, manquait d'exactitude aux yeux des Grecs. La raison était que la Grèce, à travers la voix de ses représentants politiques -à un moment de rare unanimitét ${ }^{4}$ n'acceptait guère une appellation comprenant une quelconque osmose du nom "Macédoine" (sous une forme grammaticale ou sous une autre, par exemple composée). Or, la clause européenne n'y faisait aucune allusion.

9 De plus, cette nouvelle république, venait d'adopter (11 août 1992) comme drapeau national le fameux soleil de Vergina, symbole par excellence du passé glorieux de la dynastie des anciens Macédoniens et d'Alexandre le Grand. Vergina, lieu de découverte du symbole, se trouve dans l'espace de la Macédoine grecque.

10 Pour les Grecs, c'était là à la fois une confirmation de l'usurpation de leur passé historique et culturel, et une indication de l'existence de projets irrédentistes macédoniens ${ }^{5}$. Dès lors, des milliers de Grecs de tout âge manifestent dans les rues des grandes villes, soutenus et convoqués par l'union des mairies et communes en ayant comme slogan principal : « il n'y a qu'une Macédoine et elle est grecque ».

11 Entre 1991 et 1995, des élections nationales anticipées, des manifestations de millions de Grecs, un blocus commercial à l'encontre de ce nouveau pays, une pléthore d'articles et d'émissions dans les médias ${ }^{6}$, ne sont que quelques détails d'un paysage grec fortement mouvementé. On en venait à comparer ce danger à un véritable "vol de l'âme grecque". La question de la dénomination de ce nouveau pays, fut transformée en une menace objective, mais surtout imaginaire et symbolique, pour toute une nation. 

Unies et de la Communauté européenne entre 1991 et 1995 font de cette affaire le problème prioritaire de la politique extérieure grecque et conduisent à des commentaires et à des réactions d'incompréhension, d'étonnement ou d'ironie de la part de ses partenaires sur la scène internationale.

13 À l'intérieur, le discours public (politique et médiatique) se sert de toute sa panoplie historique concernant la Macédoine. Les partenaires européens sont visés par cette propagande, mais également le public grec qui pourtant semble gagné d'avance. Dates, faits, figures et auteurs anciens sont au cœur d'une logorrhée d'un volume impressionnant ${ }^{7}$ et toute sorte de néophytes "macédonologues"8 défendent les droits de la Grèce au nom de l'histoire. Homère, Hésiode, Hérodote, Isocrate, Polybe, Plutarque renaissent de leurs cendres et la figure historique d'Alexandre le Grand est au cœur d'un passé historique glorieux revisité ad nauseam.

Un riche vocabulaire nationaliste s'est mis en place depuis 1992, surtout dans la presse'. La nouvelle république est remplacée par les mots "pseudo-État » ${ }^{10}$, « entité »,ou tout simplement « Skopje », son histoire " pseudo-histoire », ou « histoire volée et falsifiée », ses habitants « Skopjiens », ou « les Tsiganes des Balkans », ou encore, se basant sur des «textes antiques", "laids», puisque "leurs ancêtres les Dardaniens l'étaient ». La version grecque de l'histoire est baptisée " une, seule et unique vérité ", les réactions de la Grèce "nouvelle révolution culturelle de la nation $»^{11}$. À son tour, le responsable de la commission européenne M. Van der Broek est rebaptisé "Van derTurc " ${ }^{12}$, ainsi que l'affaire macédonienne qualifiée dorénavant de «skopjienne ».

Le soleil de Vergina devient symbole national par une loi votée au parlement grec (16 février 1993). De plus, son image sert de produit commercial : on la retrouve comme emblème de plusieurs établissements (par exemple, étatiques, banquiers, hôtels, moyens de transport) ainsi que sur les étiquettes de plusieurs produits de consommation. L'aéroport de Thessalonique, se rebaptise "aéroport de Macédoine" ; le port de la ville de Kavala, "Philippe II".

16 Réaction d'un peuple qui sent son identité nationale et son capital historique et symbolique menacés, et qui se défend par la revendication de ses propres origines. L'argument historique du passé antique de la région se matérialise -dans le discours public- à travers des découvertes archéologiques relativement récentes (1977) dans la région de la Macédoine grecque qui ne « laissent aucun doute ». La continuité à tous les niveaux (ethnique, culturel, linguistique, géographique) semble évidente aux yeux de ses défenseurs : la Macédoine "était depuis 3000 ans, est, et ne sera que grecque ${ }^{13}$. «L'histoire s'écrit avec du sang, elle ne se falsifie pas avec des mensonges. ${ }^{14}$ »

Un manuel d'histoire diffusé en 1992 par le ministère de l'Éducation à toutes les écoles grecques (à l'intérieur et à l'extérieur du pays), mentionnait l'historique du problème et les positions officielles du gouvernement ${ }^{15}$. Un extrait représentatif du manuel en question résume de manière "axiomatique" le problème en mettant l'accent sur la peur d'une privation identitaire :

La reconnaissance d'un État avec un nom concret donne le droit aux citoyens de cet État, et seulement à eux, de se désigner avec ce nom. Si, alors, il va être permis aux Skopjiens de désigner leur pays comme Macédoine et de se désigner eux-mêmes comme Macédoniens, nous, les Grecs macédoniens, on se prive de l'usage d'un élément de définition secondaire (à côté de "Grèce - Grecs"), qui certainement, 
nous associe à l'histoire de la Macédoine qui est d'autant plus l'histoire de l'Hellénisme.

18 Mis à part le ministère de l'Éducation, certains historiensprennent la parole : « comment les Skopjiens peuvent-ils se prétendre à la fois descendants d'Alexandre le Grand et Slaves? Il y a neuf siècles d'écart »" "Si les "Skopjiens" ont une conscience nationale grecque ils n'ont qu'à faire un référendum pour l'incorporation de leur pays à la Grèce " réclamaient certains ${ }^{17}$. Les comparaisons dans les déclarations politiques ne manquaient pas d'imagination : " les Français se sont bien disputés pour savoir si Yves Saint Laurent pouvait dénommer "Champagne" un de ses parfums, alors, nous avons bien le droit de protester contre la volonté de Skopje d'usurper le nom de Macédoine ${ }^{18}$. Ou encore, "le nom de Macédoine est comme une marque déposée. Vous, les Français, vous vous êtes bien battus pour que les marques Cognac ou Champagne ne soient pas détournées $»^{19}$.

Déjà en 1992, plusieurs rumeurs politico-médiatiques ${ }^{20}$ évoquaient dans la presse grecque une coalition entre la République de Macédoine et "l'ennemi éternel" de la Grèce, la Turquie (un peu plus tard on trouvera les États-Unis ou les "grandes puissances"), reflétant ainsi une ambiance générale de menace potentielle pour le pays. Ces menaces successives créaient un « climat d'angoisse nationale ${ }^{21}$ et alimentaient la tendance générale des Grecs à «voir partout des ennemis », une sorte de complexe national de « voisinophobie $»^{22}$.

Finalement, un accord intermédiaire signé en 1995 au siège des Nations Unies a établi les conditions nécessaires pour que le dialogue politique soit garanti. Suite à celui-ci, la République de Macédoine est amenée a changer son drapeau et certains articles dans sa constitution, et elle est officiellement acceptée aux Nations-unies, sous le nom provisoire d'Ancienne République yougoslave de Macédoine. Ce nom reste provisoire, car elle doit résoudre ce problème avec la Grèce en l'espace de sept ans de négociations.

21 Pour la plupart des pays qui l'ont reconnue officiellement elle s'appelle République de Macédoine, et sa population des Macédoniens. En Grèce, les initiales anglaises F.Y.R.O.M. ${ }^{23}$, ou encore "République de Skopje", ou simplement "Skopje", de par sa capitale, sont devenus monnaie courante et acceptables tant par l'opinion publique que par les médias et le monde politique. Suite à des accords commerciaux, la Grèce est devenue rapidement le deuxième pays le plus important en terme d'échanges économiques avec la nouvelle république.

\section{L'osmose d'un compromis}

Depuis 1995, des discussions diplomatiques répétées entre les deux pays centrées sur le seul différend du nom n'ont pas abouti à un véritable accord mais plutôt à une ambiance de rumeurs et de scepticisme. Plusieurs appellations alternatives ont été proposées $^{24}$, des solutions d'usage "œcuménique" (par tous les pays, la Grèce incluse) ou d'usage strictement "interne" à la Grèce (la thèse de la double appellation), mais tout montre que le différend concernant le nom a été renvoyé ad graecas calendas.

Un modus vivendi semblait s'instituer avec des discours politiques plutôt critiques, surtout en Grèce, par rapport au passé tumultueux de cette affaire depuis 1991. On passait à une nouvelle phase de l'affaire qu'on peut qualifier de "compromis réaliste". Suite à cette nouvelle logique, représentée par le nouveau premier ministre $\mathrm{M}$. Simitis et une bonne partie de l'opposition, la Grèce devait "en finir" avec ce problème, un de 
plus (mis à part celui de la Turquie et le problème chypriote). Des expressions du type "appellation mixte", "compromis honnête", ou "appellation composée" façonnaient la nouvelle phraséologie politique ${ }^{25}$.

Plusieurs déclarations allaient dans ce sens. À Athènes, le ministre des Affaires étrangères, M. Pangalos, affirmait que

aujourd'hui la majorité du pays est consciente, comme le montrent les sondages, qu'il faut trouver une solution. Quand on demande aux Grecs s'ils sont favorables à une solution négociée avec Skopje, ils répondent à $80 \%$ oui. Mais quand on leur demande si Skopje peut s'appeler "République de Macédoine", ils répondent à $80 \%$ non. Il faut donc résoudre ce paradoxe ${ }^{26}$

tout en demandant, un an plus tard, «moins de références à Alexandre le Grand et plus d'attention à tout ce qui se passe autour de nous $»^{27}$. À Skopje, au cours du premier entretien d'un homme politique grec avec un quotidien du pays voisin, le même ministre déclara qu'« il n'y a pas de véritable problème dans les relations entre les deux pays. L'affaire du nom est une affaire de sémantique ${ }^{28}$.

Certains membres de l'opposition soutiennent que « le nom composé vaut mieux que le nom tout court", on parle même d'une "tendance de l'opinion grecque vers un compromis ». Néanmoins, différentes réactions politiques voient le jour, comme par exemple un manifeste de 20 députés socialistes $(20$ mars 1996) et un autre de 31 députés de l'opposition (5 février 1997) quant à leur objection face à une éventuelle reconnaissance d'un nom comprenant le mot "Macédoine" ou ses composés.

De l'autre côté, le Président Gligorov évoquait dans ses déclarations, qui devenaient de plus en plus fréquentes dans les quotidiens grecs, « un énorme problème psychologique et moral » surviendrait au cas où son pays devait changer de nom ${ }^{29}$.

Les deux tiers de la population de mon pays ont toujours été des Macédoniens, ce peuple a une longue histoire et tradition, sans vouloir dire qu'ils ont quelque chose à voir avec les anciens Macédoniens...Imaginez-vous ce qui va se passer si un matin ce peuple qui a su pendant toute sa vie qu'il était Macédonien, se réveille en entendant qu'il ne l'est plus et qu'il ne parle pas la langue macédonienne ${ }^{30}$.

D'autres fois l'attention se focalise sur le présent: « nos relations actuelles comptent et non pas ce qui s'est passé il y a 3000 ans, car quand l'histoire se transpose dans la vie de tous les jours nous devenons prisonniers du passé ", ou encore "nous ne désirons pas le monopole du nom de Macédoine ", tandis que son nouveau premier ministre, M. Cervenkovski, disait que «le seul parrain d'un pays est son peuple» et qu'« en ce moment il n'y a aucune envie de changement de nom $»^{31}$.

À trois occasions les médias grecs se sont intéressés au contentieux après un long moment d'indifférence. La première, d'ordre archéologique -sinon idéologique-, concernait l'annonce de la prétendue découverte du tombeau d'Alexandre le Grand dans l'oasis de Siwa en Égypte. Une archéologue grecque, en 1995, provoqua toute une polémique spectaculaire entre la Grèce et la République de Macédoine afin de savoir,

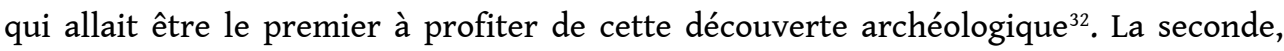
d'ordre politique -sinon psychanalytique-, relevait d'un lapsus du porte parole du gouvernement grec qui, dans une de ses déclarations aux journalistes, a "osé" prononcer l'appellation " Macédoine de Skopje » provoquant quasi automatiquement une série de réactions politiques ${ }^{33}$.

Plus récemment, la crise au Kosovo a conduit les deux pays à une collaboration étroite au niveau de leur politique extérieure face à la gestion du problème de la province 
yougoslave. Notamment lors d'une rencontre de leurs ministres des Affaires étrangères (23 juin 1998), ils ont fait appel en commun à une solution pacifique de la crise ${ }^{34}$. Un an plus tard, en pleine guerre du Kosovo, à l'occasion de l'anniversaire des cinquante ans de l'OTAN à Washington, des rumeurs journalistiques évoquaient un accord éventuel finalement non réalisé- autour d'une reconnaissance grecque du nom de «République de Macédoine-Skopje ${ }^{35}$. Il faut souligner que le contenu des articles était presque entièrement consacré aux intérêts économiques et aux investissements grecs, pendant cette phase de maturité et de sérieux des relations entre ces deux pays, parlant même d'une "occasion en or" sans faire une quelconque allusion aux relations tumultueuses du passé.

\section{Regard psycho-sociologique sur l'affaire macédonienne}

31 Le résumé de l'ambiance qui régnait en Grèce pendant cinq bonnes années, de l'indépendance à l'accord intermédiaire, nous permet de mieux comprendre le scandale provoqué par la déclaration de l'ancien premier Ministre. Comment pourrait-on évoquer l'éventuel oubli d'une affaire qui a pu rassembler des millions de Grecs dans les rues et provoquer tant d'incidents politiques et diplomatiques? Oublier reviendrait à trahir une cause nationale tant défendue.

Mais que se passe-t-il réellement aujourd'hui, cinq ans après la signature de l'accord intermédiaire? Comment les Grecs perçoivent-ils cette affaire qui reste encore insoluble ? De quoi se souviennent-ils de cette période tourmentée ? Comment gèrentils la présence de ce nouveau pays appelé désormais République de Macédoine par la grande majorité des autres pays dans le monde? Comment se représentent-ils ce pays voisin et ses habitants? Quelles ont été les répercussions de cette affaire au niveau de leur identité nationale et de la perception de leur passé historique?

Ces interrogations ont alimenté notre intérêt sur cette affaire depuis 1997. Notre problématique se développe à partir de ce cas socio-politique concret concernant un refus nominal quitouche à l'essentiel, à un des éléments fédérateurs de la culture historique et de l'identité d'un groupe national. Nous nous sommes intéressés au recours à l'histoire lointaine du groupe national, à ses fonds argumentaires et enjeux mnémoniques.

L'objectif de notre travail est de proposer une grille de lecture psycho-sociologique du refus grec, et notamment de démontrer la force du passé historique et de ses mythes dans la gestion et la construction de l'identité nationale. Ceci, en se focalisant sur l'effort grec pour justifier, à l'aide d'un argumentaire historique renvoyant à des siècles lointains, un héritage culturel et une identité régionale / nationale profondément ancrés dans la mémoire nationale historique, mais également profondément blessés par une réalité socio-politique vue, montrée, et représentée comme menaçante.

Pour cela, nous avons fait appel aux modèles théoriques des représentations ${ }^{36}$ et de la mémoire $^{37}$ sociales, afin de pouvoir donner des éléments de compréhension et d'analyse. Le domaine de recherche qui se situe autour de l'étude des représentations sociales apparaît comme "un espace privilégié pour saisir au niveau individuel et collectif, le jeu des déterminations sociales et des enjeux psychologiques dans la construction des savoirs, l'élaboration des expériences et des visions du monde social $\aleph^{38}$. Nous nous intéressons particulièrement à la lecture de l'affaire macédonienne vue par le discours public (presse grecque, manuels d'histoire), et 
surtout par le discours privé d'une population grecque, recueilli à plusieurs reprises sur le terrain (Athènes et Thessalonique) de 1997 à 1999.

Notre méthodologie combine des outils psycho-sociologiques qualitatifs (entretiens individuels et collectifs, analyse de la presse nationale et internationale, associations verbales, échelles d'attitudes, questionnaire) et la population ciblée est constituée de jeunes Grecs (de 20 à 30 ans). Elle a été sélectionnée en fonction de l'âge des sujets et de leur lieu de naissance et d'habitation (Grecs macédoniens vivant à Thessalonique versus Grecs non macédoniens vivant à Athènes), afin de mettre en jeu le facteur du lieu de naissance et de l'âge dans la perception et la représentation de cette affaire.

Dans le cadre du présent article, faute de place, nous ne présenterons qu'une partie de nos résultats. Après l'examen de certaines coupures de presse grecque et d'une partie des sondages sur les tendances de l'opinion publique envers le problème du nom, nous aborderons deux types de résultats. Le premier, issu d'une analyse de contenu qualitative de réponses à deux questions ouvertes, et le second, à partir d'un recueil d'adjectifs autour de l'image des "autres", en occurrence les habitants des pays voisins de la Grèce, mais aussi des Grecs eux-mêmes.

\section{De la « menace territoriale » au « compromis réaliste »}

Une des dimensions qui nous a frappé pendant la réalisation de notre recherche sur le terrain (Athènes, Thessalonique) tenait au fait que l'affaire, après son explosion au début des années 1990, était tombée dans les oubliettes. La plupart des jeunes interviewés parlaient franchement d'une "affaire oubliée" ; "personne n'en parle", disaient-ils souvent au cours de l'entretien, ni les médias, ni les hommes politiques, ni le milieu social ou familial proche.

\section{Trois sondages et une lettre...}

Déjà une relativisation de la menace était survenue auprès de l'opinion publique. Selon un sondage paru dans la presse en mars $1996^{39}$, moins de deux Grecs sur dix (15,7\%) considéraient l'affaire "skopjienne" comme le problème majeur de la politique extérieure grecque, contre $33 \%$ en $1995^{40}$. Cela n'est pas sans intérêt dans la mesure où, au delà du "vol d'une histoire", l'affaire macédonienne fut présentée en Grèce comme une menace territoriale objective.

Nous pouvons chercher les raisons de cette variation d'une part, dans la fin de la guerre en ex-Yougoslavie qui ne pouvait plus servir de cadre, ni d'image à ce type de menace. La fin de la guerre a également permis l'amélioration sensible des relations socioéconomiques entre les deux pays. De l'autre, la crise militaire majeure au sujet des îles Imia a réactualisé les problèmes en mer Égée entre Grèce et Turquie. Enfin, les six ans de dispute entre les deux voisins ont fatigué et surtout frustré une bonne partie de la population grecque.

41 Cette tendance de l'opinion publique est renforcée par le second résultat de cette étude, certainement le plus spectaculaire: $47,8 \%$ des interviewés considèraient «l'appellation composée $»^{41}$ comme la meilleure chose que la Grèce puisse obtenir dans ses négociations avec la République de Macédoine. 37,4\% d'entre eux étaient contre toute négociation au sujet du nom, tandis que $11 \%$ se déclaraient pour une double appellation (une pour la Grèce, une autre pour le reste des pays). Ce résultat est 
étonnant, dans la mesure où pour la première fois dans un tel sondage ${ }^{42}$, une majorité de Grecs prenait position pour une solution autre que celle du refus total et pour une négociation qui pourrait conclure à la reconnaissance d'une appellation contenant le mot "Macédoine".

Détail capital: la question posée au sujet du nom n'était pas «qu'est-ce que vous voulez? », mais « qu'est-ce qu'on peut obtenir aujourd'hui ?»

Ce détail prend tout son sens à la lumière de deux autres articles parus, juste un an après. Tout d'abord, une lettre signée par 166 élèves originaires de Thessalonique et envoyée au président de la République grecque et aux leaders des partis politiques en mai $1997^{43}$. Celle-ci semble montrer que les jeunes Grecs n'ont pas totalement oublié ce différend :

Nous sommes les Macédoniens. Les Skopjiens sont des Slaves et doivent être fiers de leur histoire slave et de leur héritage. Nous voulons vivre en paix avec nos voisins, mais nous ne pouvons pas donner notre nom. Aucune pression ne peut changer notre point de vue. Nous ne connaissons pas la politique et nous ne voulons pas la connaître.

Et maintenant, quelques questions qui demandent des réponses et non pas des manoeuvres. Voulez-vous une solution pour 1997 ou pour 2027? Est-il démocratique de décider pour nous sans nous? Nous demandez-vous d'arrêter de lire l'histoire grecque et notamment l'histoire de la lutte macédonienne? Est-il démocratique d'ignorer la volonté de la majorité du peuple grec qui est opposée à l'usage du mot "Macédoine" et de ses composés? Nous demandez vous d'être atteints d'amnésie pour que certains entrepreneurs grecs s'enrichissent en faisant des investissements à Skopje ? Nous demandez-vous d'ignorer le sacrifice de tant de Macédonomaches ${ }^{44}$ ? Allons-nous arrêter de déposer des fleurs à Pavlos Melas et au capitaine Kotta? Nous demandez-vous de ne pas nous souvenir de Melina Merkouri qui disait : « il n'y a qu'une Macédoine et celle-là est plus grecque que la Grèce »?

Nous ne connaissons pas la ou les réponses à cette lettre, mais il est évident que les élèves de Thessalonique semblaient, à l'époque, encore préoccupés par la tournure finale de cette dispute. Nous pouvons remarquer que leurs préoccupations semblent façonnées par la question du risque de confusion nominale et identitaire (localenationale), de la paix avec leur voisins, du refus de la politique et des enjeux économiques, de l'avenir, mais également de la peur d'une amnésie forcée du passé historique récent. Le ton et le style ont changé par rapport à la quantité (et la qualité) des articles consacrés à ce problème entre 1991 et 1993, néanmoins, le problème semble insoluble.

Pourtant au lendemain de la publication de cette lettre, un autre article ${ }^{45}$, dans un quotidien à grand tirage, aurait pu être considéré comme une réponse indirecte. La date est hautement symbolique puisqu'elle marque une sorte d'anniversaire de cinq ans depuis les grandes manifestations d'un million de personnes à Thessalonique. Le journal demande l'avis des 64 députés élus dans la province de Macédoine grecque au sujet de la question épineuse du nom de la république voisine. Dans leurs déclarations les députés semblent, pour la première fois et dans leur grande majorité $\left(42 / 61^{46}\right)$, abandonner la position absolue qu'ils auraient pu défendre dans le passé. Désormais, du rejet absolu d'un quelconque compromis ${ }^{47}$ au sujet du nom, on passe au credo du "réalisme politique" qui peut conduire éventuellement à un accord.

Néanmoins, à cette époque l'opinion publique ne semblait pas partager le même optimisme. Six mois plus tard, les résultats d'un sondage national, réalisé auprès d'un échantillon important de 2000 personnes à Athènes et à Thessalonique autour de cette 
même question, montrait que $77 \%$ des interviewés s'opposaient à un tel compromis ${ }^{48}$. Il faut noter que cette fois, la question concernait leur avis personnel et non pas un jugement hypothétique sur la possibilité de réussite de la politique extérieure grecque auprès de ses partenaires.

\section{Le nom et le slogan}

L'ensemble des réponses a été classé dans quatre catégonies distinctes. Néanmoins, il nous semble important de souligner que les réponses à la question du nom suscitaient une base argumentative qualitativement plus élaborée que celles du slogan. En effet, la question du nom suscitant le refus, impliquait de la part des sujets la mise en place d'une logique de justification, tandis que le slogan, lui, faisant l'objet d'un consensus positif, correspondait à une réalité de fait. En ce sens, du point de vue rhétorique, la nature des réponses à chacune des questions, c'est-à-dire la négation majoritaire (nom) et l'affirmation majoritaire (slogan), nécessite l'élaboration d'un discours d'ordre différent. La négation doit être justifiée, alors que l'affirmation renvoie à la confirmation d'une réalité, dont la certitude est si forte, que sa justification semble inutile. Nous sommes tentés de dire que la réalité exprimée par le slogan, au vu de son usage médiatique, véhicule une "vérité" idéologique profondément ancrée dans les opinions exprimées. Un exemple de réponses aux questions illustre nos propos : 


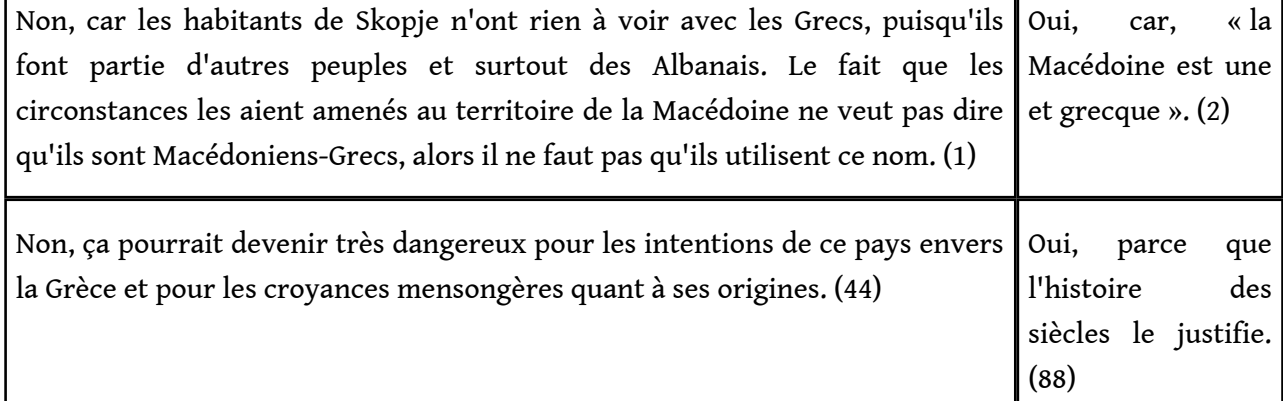

Cependant, la totalité des réponses désigne essentiellement les mêmes sources d'argumentation. Ainsi, une analyse de contenu de cette totalité nous a permis de dégager quatre catégories principales, qui seront illustrées ici par quelques réponses parmi les plus représentatives. Il s'agit de l'Histoire (33,3\% des réponses), l'Identité (30,5\%), la Politique $(24,3 \%)$ et la Géographie $(11,8 \%)$.

(a). L'histoire pour les sujets, est égale aux origines, ou plus brièvement, à la vérité. Une idée illustrée remarquablement par un sujet : « ce slogan n'a pas besoin de justification, car c'est la vérité » (38). Pour utiliser leurs mots, l'histoire est " notre origine ", à savoir "l'antiquité », c'est-à-dire "Alexandre le Grand ». La preuve ce sont les découvertes archéologiques, les sources historiques et notamment les «fouilles de Vergina, le tombeau de Philippe », mais aussi, les « manuscrits » et les « auteurs anciens».

Dans la mesure où l'histoire se matérialise à travers des "découvertes-preuves", elle devient encore plus réelle, plus indubitable. L'ancienneté lui donne une valeur de plus : c'est l'histoire "des siècles". Cependant, il ne s'agit pas d'une histoire "morte"; elle est "vivante" et en plus "agit" : elle apprend, justifie, témoigne, prouve, démontre. De la même façon, elle n'est pas un objet; ni une marchandise: elle ne peut pas "s'emprunter", ni "se vendre"; personne ne peut la "voler". "Les faits historiques ne se sont jamais référés à une Macédoine, autre que grecque " (7). La "réalité" historique aussi bien qu'une temporalité diachronique prouvent ainsi la "continuité" de l'hellénicité : "depuis 3000 ans, il y a une continuité, ainsi que des témoignages historiques qui démontrent l'hellénicité de la Macédoine » (58).

(b). Le slogan et la stéréotypie qu'il véhicule, semblent avoir fortement influencé les sujets. Leurs réponses font apparaître des traits identitaires (à savoir, origines, langue, culture, histoire, géographie) différenciateurs. La langue : « non, car leur langue et leur civilisation n'ont rien à voir avec la culture de la Macédoine » (18). L'origine nationale / ethnique : «ils ne sont pas des Grecs » (62), « ils n'ont jamais été des Grecs » (73). Le vol d'une culture : « l'appellation du lieu est correcte pour Skopje. Mais ceci vient appuyer le vol culturel de la Macédoine grecque » (26). Les liens entre identité et histoire : «je suis grecque, j'aime mon pays, je crois à l'histoire, et l'histoire dit que la Macédoine est grecque » (93). Plusieurs sujets, ne trouvent même pas la raison de se justifier au vu de la vérité du message : « oui, car elle est une et grecque, sans aucune justification » (32), ou encore «le slogan parle de lui-même » (10).

Néanmoins, quelques sujets expriment leurs réserves face au nationalisme caché des réponses "faciles et évidentes" : « elle est importante mais sans arriver aux limites du nationalisme » (24). Ou bien il montrent une certaine compréhension du problème : « il 
est vrai que les habitants de FYROM ont appris à être appelés Macédoniens depuis leur enfance » (20).

(c). Une grande partie de l'argumentation repose sur des références à la politique. Ceci, afin de montrer leur méfiance face aux gouvernements grecs : « les gouvernements ont laissé depuis longtemps écrire des choses inexactes et injustes sur la région » (28), et aux "grandes puissances" : " aucune grande puissance n'a le droit de remettre en cause un fait afin d'obtenir ses intérêts politiques et économiques » (14). Egalement, afin d'afficher leur conviction que derrière cette affaire il y a des intérêts politiques et militaires cachés : " tout ça se fait pour des raisons politiques » (19), « sous la pression de Skopje de s'appeler Macédoine se cachent des intérêts politiques et militaires » (35).

Il est nécessaire de souligner qu'une partie restreinte des réponses de cette catégorie, exprime une certaine réserve, reconnaissant un caractère nationaliste au slogan : « le slogan me parait excessivement nationaliste et très fanatique » (13), « il est nationaliste et véridique » (27), «il ne faut pas se montrer nationaliste quand il le faut (...), il est égoïste de la considérer comme territoire grec devant la peur d'une aliénation nationale » (12).

(d). La limitation du territoire de la Macédoine géographique à la partie grecque repose sur trois critères principaux. Leur propre opinion: "je suis persuadée que les frontières de la Macédoine ne s'élargissent pas hors des frontières grecques » (17). Leur opinion et leurs connaissances historico-politiques: "après la constitution de l'État grec, la Macédoine fut inclue dans les frontières grecques » (84). "Le nouvel ordre après les deux guerres mondiales a fait qu'on appelle Macédoine seulement la partie géographique de la Grèce du Nord » (66). Leur conviction : « la Macédoine appartient au territoire géographique grec, et ceci rien ne peux le changer » (95).

\section{Sources d'informations}

Nous avons voulu savoir d'où provenaient les connaissances des sujets. Pour cela, un certain nombre de questions leur était posé à la fin du fascicule. Elles étaient conçues sous deux aspects : le premier concernait la diffusion via les média (télévision, radio, journaux, magazines), le deuxième, la diffusion à travers les différents milieux de socialisation, comme le milieu institutionnel (éducation), familial ou amical, aussi bien que l'intérêt propre des sujets à se renseigner sur cette affaire. Une dernière question concernait leur orientation politique ${ }^{49}$. Les résultats de ces questions ont fait l'objet d'une analyse quantitative.

Incités plutôt par un intérêt personnel et des discussions avec leurs amis, les sujets ne mentionnent pas une influence en matière d'information et de connaissances provenant du milieu éducatif ou familial. Les résultats attestent une préférence pour des modes intensifs d'information (télévision, journaux) montrant ainsi le caractère médiatique de l'affaire. De même, ils font apparaître le caractère conjoncturel d'un événement politique privilégié par un mode d'information qui trouve appui sur un intérêt personnel, aussi que par l'échange d'informations dans le cercle amical.

\section{L'image des « autres »}

61 En 1999, dans le cadre de notre thèse, nous avons interrogé 100 autres jeunes Grecs, originaires pour moitié de Thessalonique et pour moitié d'Athènes, sur l'image qu'ils avaient des habitants des pays voisins de la Grèce (Italie, Albanie, FYROM ${ }^{50}$, Bulgarie, 
Turquie) et d'eux-mêmes (les Grecs) ${ }^{51}$. Le but de cette démarche était triple. Dans un premier temps, il s'agissait de saisir quelques traits de l'image globale des Grecs vis-àvis de leurs voisins, mais aussi d'eux mêmes, d'affiner ses structures, et de comparer certaines caractéristiques par pays. Dans un second temps, nous avons voulu analyser séparément les qualificatifs réservés aux habitants de la République de Macédoine, afin d'examiner leurs spécificités. Enfin, les résultats recueillis ont été comparés selon le facteur du lieu de naissance des sujets ${ }^{52}$. À la fin du fascicule, six questions concernant les connaissances, préférences, expériences, désirs des sujets vis-à-vis des cinq pays avoisinant la Grèce étaient proposées ${ }^{53}$.

\section{Résultats}

L'analyse du corpus des mots / expressions par pays et par population (Athènes, Thessalonique) fait apparaître trois classes lexicales. La première $(56,5 \% \mathrm{du}$ total) est réservée aux habitants d'Albanie, Turquie, FYROM et Bulgarie. La deuxième (32,9\%), regroupe les mots attribués aux habitants de l'Italie, et la troisième (10,4\%), aux habitants de la Grèce.

Les qualificatifs revenant le plus souvent pour les pays de la première classe, tant pour les sujets en provenance d'Athènes que pour ceux de Thessalonique, désignent une "image noire" attribuée aux habitants de ces pays: incultes $(49,0)$, pauvres $(37,8)$, agressifs $(34,3)$, non civilisés $(23,1)$, laids $(19,5)$, fanatiques $(17,6)$, violents $(14,1)$, sournois $(12,3)$, sous développés $(12,3)$, non hospitaliers $(10,2)$, pour ne se référer qu'à une petite partie des adjectifs qui leur sont réservés.

Ces qualificatifs ne sont pas loin de l'attribution à la grande majorité des voisins géographiquement proches de la Grèce de l'adjectif "barbares" avec tout ce que ce terme véhicule en lui d'identitaire, culturel et historique. Le syndrome de voisinophobie trouve ainsi un terrain solide, non seulement grâce à une certaine culture de la menace propre à la Grèce, mais surtout, à cause de l'univers imaginaire associé aux pays "menaçants" et à leurs habitants.

À la suite de notre analyse, le contraste est encore plus frappant à la lumière du haut degré positif des adjectifs qualifiant, non seulement les Grecs : ont du zèle (philotimoi) $(37,9)$, bon vivants $(26,8)$, sympathiques $(18,3)$ et patriotes $(18,1)$, mais aussi les Italiens : romantiques $(39,8)$, beaux $(33,1)$, vivants $(33,1)$, cultivés $(28,7)$, civilisés $(14,7)$, hospitaliers $(11,6)$. Il est sans doute rare, sinon antinomique -toute une tradition de recherche en psychologie sociale en témoigne ${ }^{54}$ - de ne pas qualifier son propre groupe national (et donc soi-même) positivement. Toutefois, force est de constater que les qualificatifs concernant les Italiens ont une double fonction : ils servent tantôt d'antonymes (par exemple beaux versus laids, cultivés versus incultes), vis-à-vis des habitants des autres pays, tantôt de synonymes (par exemple ouverts - bons vivants, gentils - chaleureux) face aux adjectifs réservés aux Grecs. La connotation identitaire de ces effets sémantiques n'est pas anodine, car elle manifeste une connotation identitaire positive envers les Italiens, aussi bien qu'une différenciation marquante entre ces deux groupes nationaux et le reste.

\section{Un groupe à part}

Un regard plus attentif aux adjectifs / expressions retenus pour les habitants de la République de Macédoine n'est pas sans surprise. Une comparaison du vocabulaire en 
provenance des deux villes (Athènes et Thessalonique) avec la totalité des mots / expressions utilisés pour les autres pays, a mis en lumière 28 traits spécifiques, utilisés exclusivement pour caractériser ces habitants :

Vocabulaire spécifique antipathiques (2), balkaniques (2), bâtards, confus, frustrés, hétérophotes $(2)^{55}$, ils essayent de prendre quelque chose qui ne leur appartient pas,

ils ont adopté notre nom et notre histoire, indifférents quant aux droits nationaux des autres, inexistants, injustes, fauteurs de troubles, insistants, mal informés, minorité qui revendique à tort, moins que rien, mythomanes (2), non macédoniens (2), ont du toupet (3), primitifs (2), revendicateurs de choses étrangères à eux, sans connaissance de l'histoire (26), sournois, usurpateurs de la patrie, usurpateurs des monuments antiques, victimes de leur imagination, voleurs de civilisation, voleurs de l'histoire. mêmes habitants ("mythomanes ", "primitifs ", "bâtards ", " antipathiques ", " confus », «frustrés »). Ce mécanisme socio-cognitif d'attribution de traits de nature psychologique à autrui (individu ou groupe) est connu en psychologie sociale, sous le nom de psychologisme. Il consiste en une organisation de la perception d'autrui, qui prend forme dans la société, "selon un mode monolithique, conventionnel et profondément normatif qui explique tout ce qui échappe à la norme sociale comme non objectif, déséquilibré, voire anormal $\aleph^{57}$. Ceci n'est pas sans nous rappeler l'influence du vocabulaire de la presse ${ }^{58}$ (laids, Tsiganes des Balkans) pendant la période explosive de l'affaire dans la première moitié des années 1990, aussi bien que l'axiomatique du manuel d'histoire.

Les qualificatifs réservés aux habitants de la République de Macédoine s'insèrent parfaitement au sein des représentations barbares des habitants des autres pays. Leur particularité, tant au niveau de leur degré de négativité qu'à celui du vocabulaire spécifique, renvoie à des significations profondément marquées par l'influence médiatique et institutionnelle, ainsi que par le souvenir des événements récents qui ont laissé aux Grecs un parfum d'injustice et de frustration. Ce constat se trouve également 
renforcé par le fait que seulement trois sujets sur 100 avaient déjà visité le pays en question et aucun ne s'est prononcé en faveur d'une éventuelle visite.

Selon Tajfel ${ }^{59}$, la construction des stéréotypes sociaux est à la base d'un processus de catégorisation et de différenciation avec l'autre. La population interrogée semble avoir interiorisé et partagé tout un système de stéréotypes sociaux concernant ses voisins. Leurs traits différenciateurs sont accentués à un tel niveau d'intensité, que toute peur d'assimilation avec l'autre est minimisée. Les habitants de la République de Macédoine ne font pas exception à la règle. Leur traits sont ancrés dans l'image négative des autres, tandis que leurs particularités tiennent au différend entre les deux pays.

Plusieurs études en psychologie sociale insistent sur le lien entre représentations et structures sociales. Moscovici souligne qu'une représentation associe toujours une forme cognitive d'expression à un contenu largement accepté par le groupe ${ }^{60}$. Le précédent de la crise majeure entre les deux pays en relation avec une vague de surpublication de la presse, d'articles majoritairement agressifs envers la République de Macédoine, et ethnocentriques envers la Grèce ${ }^{61}$, ont influencé une bonne partie des représentations actuelles des Grecs vis-à-vis de leurs voisins. Nos résultats montrent ainsi le conformisme manifeste de ces caractérisations tant par rapport à la norme du groupe national, que par le système de valeurs d'une société entière.

\section{En guise d'épilogue}

72 Épilogue relatif puisque le contentieux entre les deux pays continue d'une manière latente au moment où ces lignes sont écrites. Plusieurs vagues de cette affaire sont venues bouleverser le paysage politique et social grec devenant l'un des événements majeurs de l'histoire du pays depuis la fin de la dictature des colonels.

Parallèlement, cette affaire s'inscrit dans la suite des épiphénomènes directs liés à la disparition de la Yougoslavie qui a laissé des traces tragiques et traumatiques dans les Balkans. Les changements politiques dans la région ont, à leur tour, suscité une nouvelle vision du passé historique de chaque population forcément plus centrée sur elle même. Une histoire nationale unique et unificatrice (celle de la Yougoslavie) s'est transformée en unités de mémoire nationale distinctes et antagonistes. La réécriture de chaque histoire nationale a conduit à la surenchère de la diachronie des conflits intercommunautaires, pour justifier ainsi un nouveau présent et surtout un tout nouvel avenir.

74 La prophétie de l'oubli, que nous évoquions au début de cet article, n'est finalement vraie qu'en partie. Il est certain que, depuis maintenant quatre ans, les médias grecs ont "oublié" cette affaire et que l'évolution des relations diplomatiques et économiques entre les deux pays n'a pas été freinée par le différend sur le nom. Néanmoins, malgré les différences nettes des sondages entre le pouvoir et le vouloir de l'opinion publique et de la classe politique, nos résultats démontrent que le représenter demeure essentiel. Au delà de sa simple non-évocation médiatique actuelle, le contentieux macédonien a laissé des traces multiples latentes dans la mémoire de la population grecque et a préparé le terrain pour la formation de représentations sociales de l'autre marquées par l'agressivité primaire, la polémique, sinon la frustration.

De l'hystérie collective au réalisme politique, l'imbroglio macédonien soulève des questions identitaires épineuses où la gestion du passé historique collectif tient le premier rôle. Un passé vécu, écrit, appris, nommé, revendiqué et subi de manière 
parfois radicalement différente au sein d'une même population nationale. Un passé qui réémerge et redevient objet de disputes et de guerres, mais aussi de partage de cultures, d'échange, de proximité humaine. Un passé énigmatique.

\section{NOTES}

1. Dès l'époque des successeurs d'Alexandre le Grand, la Macédoine s'élargit, et devient un consensus géographique dénationalisé sous la forme de "royaume", "éparchie", "thème", ou "vilayet". Ces notions décrivent une région administrative, aux frontières indéterminées, ayant appartenu successivement aux empires des Romains, des Byzantins et des Ottomans. Ce flou géographique posait déjà des problèmes aux géographes et aux voyageurs du XIX ${ }^{\text {ème }}$ siècle(Pouqueville (François C.), Voyage de la Grèce, Paris, 1826 ; Mannert (Konrad), Géographie des Grecs et des Romains, Landshut, 1812). La notion géographique de "Macédoine", telle qu'on la connaît de nos jours, a un sens conventionnel qui lui a été attribué par la diplomatie et l'histoire du XIX ${ }^{\text {ème }}$ siècle (Lory (Bernard), "Approches de l'identité macédonienne ", in Chiclet (Chistophe), Lory (Bernard), éds, La République de Macédoine, Paris: Ll'Harmattan, 1998). Malheureusement, faute de place, nous ne pouvons pas consacrer dans le cadre de cet article une partie sur le passé historique tumultueux de l'imbroglio macédonien depuis la fin du XIX ème siècle.

2. "The Republic cares for the status and rights of those persons belonging to the Macedonian people in neighbouring countries, as well as Macedonian expatriates, assists their cultural development and promotes links with them", article 49, paragraphe 1 de la Constitution de la République de Macédoine (1991). Sources : Ministère de l'Information RM.

3. Valinakis (G.), Dalis (S.), eds, L'affaire de Skopje. Documents Officiels, 1990-1996, Athènes: ELIAMEP, 1996, pp. 51-52, et aussi The Independent, 04/01/92.

4. C'était en effet la première rencontre des leaders des partis politiques grecs sous l'égide du Président de la République (18/02/92) et (13/04/92). Leur décision fut unanime, à l'exception du parti communiste.

5. Prevelakis (Georges), « Querelle byzantine », Le Monde Diplomatique, janvier 1999, p. 9.

6. Armenakis (Antonis) et al., «Le nationalisme dans la presse grecque. L'affaire macédonienne entre décembre 1991-avril 1993 ». The Greek Review of Social Research, (89-90), 1996.

7. Entre décembre 1991 et avril 1993 environ 2000 articles ont été publiés à ce sujet. Une étude exhaustive effectuée par des sociologues grecs corrobore nos propos (ibid.).

8. Kofos (Evangelos), «Greece's Macedonian Adventure: The Controversy over FYROM's Independence and Recognition ", in Coufoudakis (Van), Psomiades (Harry J.), Gerolymatos (Andre), eds, Greece and the New Balkans: Challenges and Opportunities, New York: New York and Pella Publishing Company, 1999.

9. Demertzis (Nicolas), Papathanassopoulos (Stylianos), Armenakis (Antonis), « Media and Nationalism . The Macedonian Question ", Press / Politics, 4 (3), 1999.

10. Notons au passage que ce même qualificatif (pseudo-État) est utilisé en Grèce depuis 1974 uniquement pour parler du territoire chypriote occupé par les forces militaires turques. Le parallèle n'est pas anodin du point de vue de la mémoire historique grecque.

11. Niki, 26/04/92. 
12. Chiclet (Christophe), «Pourquoi la Grèce a peur de la Macédoine », in Chiclet (Christophe), Lory (Bernard), éds, op.cit., p. 96.

13. Ta Nea, $13 / 05 / 92$.

14. Eleutheros typos, 06/05/92.

15. Macédoine, Histoire et Politique, Athènes : Ministère de l'Éducation et des Religions, 1992.

16. Tsirkinidis (H.), Le Monde diplomatique, mai 1994.

17. Kazanas (K.), «L'historien Polybe à propos de la Macédoine », Nea Estia, (numéro spécial sur la Macédoine), noël 1992.

18. K. Daskalaki, tête de liste -à l'époque-, aux élections européennes du parti "Printemps Politique" de A. Samaras (Le Monde, « Athènes et les Onze : la double méprise », 25/06/94).

19. N. Mertzos, directeur de l'hebdomadaire salonicien Ellenikos Voras (propos recueillis par Chiclet (Christophe), art.cit., p. 95).

20. To Vima, 22/11/92; Oikonomikos Tachydromos, 19/11/92 (cités in Roudometof (Victor), "Nationalism and Identity Politics in the Balkans: Greece and the Macedonian Question», Journal of Modern Greek Studies, 14 (2), 1996.

21. Panagiotopoulou (Roy), "Greeks in Europe : Antinomies in National Identities ", Journal of Modern Greek Studies, 15 (2), 1997, p. 357.

22. Chiclet (Christophe), art.cit., p. 97 ; Roudometof (Victor), art.cit. p. 259.

23. Former Yougoslav Republic of Macedonia.

24. Nous soulignons quelques-unes mentionnées dans la presse grecque : "Nouvelle Macédoine", "Macédoine du Nord", "Macédoine slave", "Nova Macedonia", "Macédoine-Skopje".

25. Expressions relevées par un nombre important d'articles de quotidiens grecs à large distribution (To Vima, Eleutherotypia, Ta Nea, Kathimerini).

26. Pangalos (Théodore), « La Grèce et ses conflits en ex-Yougoslavie », Confluences Méditerranée,

(14), printemps 1995.

27. Eleutherotypia, 13/03/96.

28. Dnevnik, 11/04/98, Eleutherotypia, 13/04/98.

29. Eleutherotypia, 15/06/96.

30. Eleutherotypia, 05/11/97.

31. Eleutherotypia, $12 / 05 / 97$ et 30/06/97.

32. Le Monde, "L'annonce de la découverte du tombeau d'Alexandre serait une surenchère ", 07/02/95, et Chauveau (C.), «Les États utilisent l'archéologie à leur profit », Le Monde, 25/03/95. To Vima, 19/02/95.

33. Ta Nea, 05/03/98.

34. Samary (Catherine), "Veillée d'armes au Kosovo », Le Monde diplomatique, septembre 1998, p.10.

35. To Vima, 10/04/99, 18/04/99. Eleutherotypia, 11/04/99.

36. Moscovici (Serge), La psychanalyse, son image et son public, Paris : PUF, 1961 ; Jodelet (Denise), éd., Les représentations sociales, Paris : PUF, 1989.

37. Halbwachs (Maurice), La mémoire collective, Paris : Albin Michel, 1997 [1950].

38. Jodelet (Denise), « Pensée sociale et historicité », Psychisme et histoire, 8 (1-4: « Technologies, Idéologies, Pratiques »), 1988, p. 397.

39. Sondage Alco-Martel, Eleutherotypia, 17/03/96.

40. La grande majorité des interviewés $(73,4 \%)$ identifiait les relations gréco-turques comme problème majeur.

41. Voire appellation contenant sous une forme grammaticale quelconque (adjectif et / ou nom), le mot "Macédoine".

42. Résultats proportionnels aux électeurs des deux plus grands partis de la scène politique grecque (socialiste, droite). Les électeurs de la gauche et du P.C. grec sont majoritairement plus enclins à une appellation composée. Les électeurs du "Printemps politique", parti de droite ayant 
fait de l'affaire macédonienne son cheval de bataille, sont à $31,3 \%$ positifs à l'appellation composée.

43. Ta Nea, 17/05/99.

44. "Macédonomaches": appellation des soldats grecs qui se sont battus pour la cause macédonienne (1904-1908).

45. Eleutherotypia, 18/05/97.

46. Trois députés n'ont pas répondu. En effet l'auteur de l'article distingue trois catégories de réponses. Celles qui sont catégoriquement contre un compromis (19/61), celles qui sont positives sous conditions (22/61) et celles "réalistes", qui, sans dire "oui" à une appellation composée, ne la réfutent pas non plus complètement (20/61). Il faut souligner le fait que l'article contient les déclarations signées de tous les députés interviewés.

47. Il faut peut être souligner que "compromis" dans ce cas précis signifie pour les Grecs l'acceptation d'une appellation composée.

48. Parmi eux $61,9 \%$ disaient "catégoriquement non", $14,9 \%$ "probablement non", 11,4 \% "probablement oui", 6,3\% “catégoriquement oui" et 5,5\% "ne savent pas / ne répondent pas". Ce sondage ne souligne pas de différence majeure entre l'échantillon d'Athènes et celui de Thessalonique. Sondage réalisé par la société MRB. Nikolakopoulos (H.), Communication au colloque Politique extérieure grecque et médias, Thessalonique, novembre 1997, in Ta Nea, 29/11/97.

49. La moitié des sujets n'ayant pas voulu prendre de position sur cette question, les résultats ne peuvent pas être considérés comme significatifs.

50. C'est sous ce nom, officiellement reconnu en Grèce, que la République de Macédoine était présentée aux sujets.

51. Il s'agissait d'une population d'étudiants. L'étude était censée faire partie d'une recherche européenne sur l'image des habitants des pays voisins. Les sujets indiquaient aussi, sur une échelle en cinq points, l'attribution d'un degré de positivité et de négativité à leurs adjectifs.

52. Les trois temps de cette analyse ont été assistés par un outil informatique d'analyse lexicale (Alceste).

53. En résumé, les questions étaient les suivantes : «le(s)quel(s) des pays suivants (Albanie, Bulgarie, FYROM, Italie, Turquie) : connaissez-vous le mieux, préférez-vous, avez-vous déjà visité, voulez-vous visitez, considérez-vous comme le plus agressif / le plus amical ?».

54. Tajfel (Henri), Human groups and social categories: studies in Social Psychology, Cambridge: Cambridge University Press, 1981 ; Billig (Michael), Banal Nationalism, London : Sage, 1995.

55. Adjectif grec, difficile à traduire en français. Une définition relativement proche serait : ceux qui n'ont pas fait l'effort d'apprendre par eux-mêmes, qui ont utilisé les connaissances des autres afin d'évoluer, de se constituer comme tels (du grec hétéro, l'autre, et phos, lumière, savoir).

56. Les sujets d'Athènes utilisent exclusivement cette expression pour FYROM, tandis que les sujets de Thessalonique l'utilisent majoritairement pour ce pays (39 fois au total, 22 fois pour FYROM).

57. Maggi (J.), Mugny (G.), Papastamou (S.), «Les styles de comportement et leur représentation sociale ", in Moscovici (Serge), éd., Psychologie Sociale, Paris : PUF, 1998 [1984].

58. À titre illustratif, la "une" d'un quotidien grec (de droite) : «Nous et les barbares de l'Europe », Eleutheros typos, 14/06/92.

59. Tajfel (Henri), op.cit.

60. Moscovici (Serge), « Notes towards a description of social representations ", European Journal of Social Psychology, 18 (3), 1988.

61. Panagiotopoulou (R.), « Construction des stéréotypes ethnocentriques par la presse grecque à partir de l'affaire macédonienne », The Greek Review of Social Research, (89-90), 1996. 


\section{AUTEUR}

NIKOS KALAMPALIKIS

Laboratoire de Psychologie Sociale, École des Hautes Études en Sciences Sociales (Paris). 